\title{
Ionic Binding in a Susy Background
}

\author{
L. Clavelli* and T. Lovorn ${ }^{\dagger}$ \\ Department of Physics and Astronomy \\ University of Alabama \\ Tuscaloosa AL 35487
}

October 19, 2018

\begin{abstract}
From string theory and the observation of a positive vacuum energy in our universe it seems inevitable that there will eventually be a phase transition to an exactly supersymmetric (susy) universe. In this phase there will be an effective weakening of the Pauli principle due to fermi-bose degeneracy. As a consequence molecular binding will be significantly affected. We make some general comments on susy molecules and perform a variational principle estimate of ionic binding energies.
\end{abstract}

\section{Introduction}

Very little is known at present about the behavior of bulk matter in a supersymmetric background. Since supersymmetry is a prominent feature of string vacua a prime question is whether or not the evolution of intelligent supersymmetric life forms would be possible in such a background. If not, since we could hardly expect to be making observations in a universe which did not support life, observer bias would offer some type of explanation for why our universe has, or seems to have, a broken supersymmetry (susy). In this sense a fuller understanding why our universe is as it is might require an exploration of alternative universes.

A supersymmetric universe, at least in flat space, would be expected to have a lower vacuum energy than the observed positive vacuum energy of our current universe. Therefore, from the string theory point of view, the current universe is intrinsically unstable [1]

*lclavell@bama.ua.edu

†tflovorn@bama.ua.edu 
to a future exactly supersymmetric universe with, possibly, zero vacuum energy. A first phenomenological glance at the properties of such a universe has been already undertaken [2]. As discussed there the transition will begin with the quantum nucleation of a bubble of true (susy) vacuum of radius sufficiently large to allow bubble growth. It is likely that this critical size is of galactic dimensions [3]. As the scale of the universe expands, the probability per unit time for the nucleation of a critically sized bubble will grow without limit until the transition occurs. If the current acceleration continues until all matter is trapped in white dwarfs, neutron stars, and black holes, the eventual phase transition will lead to a rapid series of gamma ray bursts [4] followed by collapse of all matter into susy black holes [5]. On the other hand, if the transition occurs soon enough that earth-like planets are still orbiting stars, susy planets will be formed and it is possible, depending on susy molecular physics, that susy life forms will evolve [2]. The modest goal of the current paper, however, is solely a study of the properties of ionically bound molecules assuming susy atoms come into existence in the form of small, heavy nuclei surrounded by an electron/selectron cloud.

In our broken susy universe, the structure of matter is dominated by the Pauli principle which forces bound fermions into high energy levels. After a transition to the exact susy universe, these excited fermions will convert in pairs to their degenerate scalar partners through gluino or photino exchange:

$$
f f \rightarrow \tilde{f} \tilde{f}
$$

The scalars, $\tilde{f}$, not being constrained by the Pauli principle, will fall into the ground state wave function via, for example, photon emission. Thus, in exact susy, there will be no more than two fermions of each type in each bound state. The remaining baryon and lepton number will be filled out by sbaryons and sleptons with all stable nuclei and atoms containing particles in s-waves only. Where possible without confusion, we will refer generically to fermionic and scalar electrons as electrons and to fermionic and scalar nucleons as nucleons. In exact susy all particles and all bound states are members of degenerate susy multiplets.

Our variational approach allows an approximate but analytic solution for the ion energies, mean radii, and ionic binding energies. For the sake of numerical comparison with our world, we evaluate our formulae in the case that the masses of the degenerate susy particles will be the masses of the standard model particles in the broken susy world. This is approximately what would be expected if the ground state of the universe corresponded to one of the higgs extensions of the minimal supersymmetric standard model (MSSM) with all susy breaking parameters set to zerd*. In these models the coupling constants would also be expected to be approximately as they are in the broken susy world with small deviations given by the running of the couplings over a slightly larger domain of exact susy. Other models for susy breaking in our world, such as gauge mediated models, could lead to similar results as the susy breaking is turned off. In the model of radiative breaking of electroweak symmetry, the symmetry breakdown of the standard model is due to a squared Higgs mass running to negative values. This could continue to be true if exact supersymmetry extends into the low energy domain

\footnotetext{
${ }^{*}$ S. Nasri, private communication
} 
One could also entertain alternative possibilities for the ground state of the universe such as the supersymmetric AdS/CFT model or one of the five flat space superstring theories. These might qualitatively share some of the features we investigate here. We give analytic results for the energies and mean radii, so the consequences of other assumptions about the degenerate susy multiplet masses and charges can be readily evaluated.

In section II we approximate the multi-electron wave function as products of hydrogenic wave functions. This wave function is improved by the variational method to take into account screening in section III. In section IV we calculate the ionic bonding of some diatomic molecules that are prominent in our broken susy world and in section $\mathrm{V}$ we treat ionic binding of the susy water molecule. We conclude with a summary and some discussion in section VI.

\section{Susy ions}

Working in natural units, $\hbar=c=1$, the Hamiltonian for the system of nuclear charge $Z$ and $N$ electrons is given by:

$$
H=-\frac{1}{2 m} \sum_{i=1}^{N} \nabla_{i}^{2}-Z e^{2} \sum_{i=1}^{N} \frac{1}{r_{i}}+e^{2} \sum_{i<j}^{N} \frac{1}{\left|\mathbf{r}_{\mathbf{i}}-\mathbf{r}_{\mathbf{j}}\right|}
$$

with $e^{2}$ being the fine structure constant, $\alpha \approx 1 / 137$, and $m$ being the electron mass.

From the variational principle, an upper bound on the ground state energy is given by the expectation value of the Hamiltonian for any trial wavefunction.

$$
E_{g s}=\langle\psi|H| \psi\rangle
$$

The ground state wavefunction for the hydrogen atom is given by:

$$
\psi_{100}=\sqrt{\frac{\left(m e^{2} Z\right)^{3}}{\pi}} e^{-m e^{2} Z r} .
$$

We might take the trial wavefunction for the general ion to be the product of this ground state wavefunction for each electron.

$$
\psi=\left(\frac{\left(m e^{2} Z\right)^{3}}{\pi}\right)^{N / 2} e^{-m e^{2} Z \sum r_{i}}
$$

This is an eigenfunction of the first two terms in eq.2.1 with eigenvalue

$$
\langle H\rangle_{\text {Hydrogenic }}=-R_{\infty} Z^{2} N
$$

where $R_{\infty}$ is the Rydberg constant

$$
R_{\infty}=m e^{4} / 2=13.6 e V
$$


This wave function is exact for the hydrogenic atoms $(N=1, Z$ arbitrary) ignoring nuclear effects and relativistic corrections. For $N>2$ fermionic electrons, this would not be expected to be a good approximation since only two electrons can be in this $1 s$ wave function. However, in the exact susy world, all of the charged leptons can be in the ground state wave function with as many of them as necessary becoming bosons via the pair conversion process of eq.1.1. Note that the conversion process occurs generically in susy and does not require $R$ parity violation.

Now, we need to calculate the expectation value of the last term, corresponding to the repulsion between the electrons

$$
V_{e e} \equiv e^{2} \sum_{i<j}^{N} \frac{1}{\left|\mathbf{r}_{\mathbf{i}}-\mathbf{r}_{\mathbf{j}}\right|}
$$

We calculate $\left\langle V_{e e}\right\rangle$ directly by integration.

$$
\left\langle V_{e e}\right\rangle=e^{2} \int \psi^{2}\left(\sum_{i>j}^{N} \frac{1}{\left|\mathbf{r}_{\mathbf{i}}-\mathbf{r}_{\mathbf{j}}\right|}\right) d \mathbf{r}_{\mathbf{1}} d \mathbf{r}_{\mathbf{2}} d \mathbf{r}_{\mathbf{3}} \ldots d \mathbf{r}_{\mathbf{N}}
$$

The number of terms in the sum is equal to $\left(\begin{array}{c}N \\ 2\end{array}\right)$. By symmetry, this integral can be rewritten and simplified.

$$
\left\langle V_{e e}\right\rangle=e^{2}\left(\begin{array}{c}
N \\
2
\end{array}\right)\left(\frac{\left(m e^{2} Z\right)^{3}}{\pi}\right)^{2} \int e^{-2\left(m e^{2} Z\right)\left(r_{1}+r_{2}\right)}\left(\frac{1}{\left|\mathbf{r}_{\mathbf{1}}-\mathbf{r}_{\mathbf{2}}\right|}\right) d \mathbf{r}_{\mathbf{1}} d \mathbf{r}_{\mathbf{2}}
$$

To evaluate this integral, we set the coordinates relative to $\mathbf{r}_{\mathbf{1}}$, so that the angle between $\mathbf{r}_{1}$ and $\mathbf{r}_{2}$ is simply $\theta_{2}$. By the law of cosines,

$$
\left|\mathbf{r}_{\mathbf{1}}-\mathbf{r}_{\mathbf{2}}\right|=\sqrt{r_{1}^{2}+r_{2}^{2}-2 r_{1} r_{2} \cos \theta_{2}}
$$

We start by evaluating with respect to $\mathbf{r}_{\mathbf{2}}$, defining a new integral:

$$
I_{2} \equiv \int e^{-2\left(m e^{2} Z\right) r_{2}} \frac{1}{\sqrt{r_{1}^{2}+r_{2}^{2}-2 r_{1} r_{2} \cos \theta_{2}}} r_{2}^{2} \sin \theta_{2} d r_{2} d \theta_{2} d \phi_{2}
$$

The $\phi_{2}$ integral is just $2 \pi$. The $\theta_{2}$ integral is a bit more involved:

$$
\begin{aligned}
\int_{0}^{\pi} \frac{\sin \theta_{2}}{\sqrt{r_{1}^{2}+r_{2}^{2}-2 r_{1} r_{2} \cos \theta_{2}}} d \theta_{2} & =\left[\frac{-1}{r_{1} r_{2}} \sqrt{r_{1}^{2}+r_{2}^{2}-2 r_{1} r_{2} \cos \theta_{2}}\right]_{0}^{\pi} \\
& =\frac{1}{r_{1} r_{2}}\left(r_{1}+r_{2}-\left|r_{1}-r_{2}\right|\right) .
\end{aligned}
$$

This is $2 / r_{1}$ for $r_{2}<r_{1}$ and $2 / r_{2}$ for $r_{2}>r_{1}$. 
Inserting these back into $I_{2}$, we get the following:

$$
\begin{aligned}
I_{2} & =4 \pi\left(\frac{1}{r_{1}} \int_{0}^{r_{1}} e^{-2\left(m e^{2} Z\right) r_{2}} r_{2}^{2} d r_{2}+\int_{r_{1}}^{\infty} e^{-2\left(m e^{2} Z\right) r_{2}} r_{2} d r_{2}\right) \\
& =\frac{\pi}{\left(m e^{2} Z\right)^{3} r_{1}}\left(1-\left(1+m e^{2} Z r_{1}\right) e^{2 m e^{2} Z r_{1}}\right)
\end{aligned}
$$

Returning to $\left\langle V_{e e}\right\rangle$, we now have:

$$
\begin{aligned}
\left\langle V_{e e}\right\rangle & =e^{2}\left(\begin{array}{c}
N \\
2
\end{array}\right) 4\left(m e^{2} Z\right)^{3} \int_{0}^{\infty} e^{-2 m e^{2} Z r_{1}} r_{1}\left(1-\left(1+m e^{2} Z r_{1}\right) e^{-2 m e^{2} Z r_{1}}\right) d r_{1} \\
& =\left(\begin{array}{c}
N \\
2
\end{array}\right) \frac{5 R_{\infty} Z}{4} .
\end{aligned}
$$

Including the hydrogen-like term from eq.2.4, we have

$$
\langle H\rangle=-R_{\infty} Z N\left(Z-\frac{5}{8}(N-1)\right)
$$

By the variational principle this gives an upper limit to the ground state energy or a lower limit to the ground state binding energy.

If $N=Z$,

$$
\langle H\rangle=-\frac{R_{\infty}}{8}\left(3 Z^{3}+5 Z^{2}\right)
$$

\section{A Better Approximation}

We can improve this upper bound anticipating that electron shielding of the nuclear charge will reduce the coefficient in the wave function exponential. With $Z_{s}$ a dimensionfull parameter to be determined by energy minimization we take the normalized trial wavefunction for the $N$ electrons to be:

$$
\psi=\prod_{i=1}^{N}\left(\sqrt{\frac{Z_{s}^{3}}{\pi}} e^{-Z_{s} r_{i}}\right) \quad .
$$

Proceeding as in section 2, we can write the expectation values

$$
\begin{gathered}
\left\langle\frac{1}{r_{i}}\right\rangle=4 \pi \int_{0}^{\infty}\left(\frac{Z_{s}^{3}}{\pi}\right) e^{-2 Z_{s} r} r d r=Z_{s}, \\
\left\langle\nabla_{i}^{2}\right\rangle=\left\langle\frac{d^{2}}{d r_{i}^{2}}+\frac{2}{r_{i}} \frac{d}{d r_{i}}\right\rangle=-Z_{s}^{2},
\end{gathered}
$$


and

$$
\left\langle\frac{1}{\left|\vec{r}_{i}-\vec{r}_{j}\right|}\right\rangle=\frac{5}{8} Z_{s}
$$

Thus

$$
\langle H\rangle=N \frac{Z_{s}^{2}}{2 m}-Z e^{2} Z_{s} N+\frac{N(N-1)}{2} e^{2} \frac{5}{8} Z_{s}
$$

This is a minimum at

$$
Z_{s}=m e^{2}\left(Z-\frac{5}{16}(N-1)\right)
$$

Since $Z_{s}$ must be positive in order to have a normalizeable wave function, this equation is only meaningful for

$$
N<\frac{16}{5} Z+1
$$

Putting this back into the expression for $\langle H\rangle$ gives

$$
\langle H\rangle=E(N, Z)=-R_{\infty} N\left(Z-\frac{5}{16}(N-1)\right)^{2} .
$$

Comparing with eq.2.14, we see that the ground state energy estimate is reduced by the improved wave function providing a decreased upper limit to the actual energy and an increased lower limit, $-E(N, Z)$, to the actual binding energy. According to the variational principle, further improvements in the trial wave function will yield a binding energy approximation approaching the exact solution from below. This variational result was known shortly after Schroedinger's original result [6] and improves the accuracy of the theoretical energy of the helium-like atoms $(N=2, Z$ arbitrary) by a factor of three above the result based on treating the electron repulsion perturbatively [7].

Beyond $N=2$ the result is not a useful approximation in the broken susy world due to the Pauli principle which forbids more than two electrons from occupying the $1 s$ level. With the circumvention of the Pauli principle in the exact susy case, we can expect comparable accuracy for susy atoms of arbitrary $N$ as for standard helium.

The average distance between an electron and the nucleus is

$$
r(N, Z)=4 \pi \frac{Z_{s}^{3}}{\pi} \int_{0}^{\infty} r^{3} d r e^{-2 Z_{s} r}=\frac{3}{2 Z_{s}}=\frac{3 \alpha}{4 R_{\infty}}\left(Z-\frac{5}{16}(N-1)\right)^{-1} .
$$

except that for $N=0$ we must put $r(N, Z)=0$.

We see that, at fixed $Z$, this mean distance is increased over the hydrogenic $(N=1)$ case due to the electron repulsion. The size of the neutral atoms $(N=Z)$ decreases rapidly for increasing $Z$ unlike the case in the broken susy world as discussed below. However, at least for moderate $Z$, the mean radius of the electron cloud remains large compared to the nuclear size. 


\section{Ionic Binding}

Ionic binding occurs when it is energetically advantageous to transfer one or more electrons from one atom to another after which the positive and negative ions strongly attract each other. Ionic binding, when appreciable, tends to dominate over covalent binding which corresponds to two atoms sharing electrons equally. In the broken susy world, ionic binding is most prominent in the alkali-halide compounds where one electron can be taken from an outer shell of one atom and used to form a closed shell on the other atom.

The change in energy after transferring $l$ electrons/selectrons from one ion of atomic number $Z_{1}$ to another of atomic number $Z_{2}$ and taking into account the subsequent Coulomb attraction is

$$
\begin{aligned}
\Delta E=-E\left(N_{1}, Z_{1}\right)-E\left(N_{2}, Z_{2}\right) & +E\left(N_{2}+l, Z_{2}\right)+E\left(N_{1}-l, Z_{1}\right) \\
& -\frac{e^{2} l^{2}}{r\left(N_{1}-l, Z_{1}\right)+r\left(N_{2}+l, Z_{2}\right)} .
\end{aligned}
$$

The negative of $\Delta E$ is the molecular binding energy. This expression assumes that the perturbation of the atomic wave functions away from spherical symmetry can be neglected and that the interatomic distance is rougly the sum of the radii of the two ions. These approximations are reasonable as a first attempt to estimate molecular properties in an exact susy background.

Assuming only single electron transfer, The ionic molecular binding energy for two neutral atoms of atomic numbers $Z_{1}$ and $Z_{2}$ is

$$
\begin{array}{r}
B=+E\left(Z_{1}, Z_{1}\right)+E\left(Z_{2}, Z_{2}\right)-E\left(Z_{2}+1, Z_{2}\right)-E\left(Z_{1}-1, Z_{1}\right) \\
+\frac{e^{2}}{r\left(Z_{2}+1, Z_{2}\right)+r\left(Z_{1}-1, Z_{1}\right)} .
\end{array}
$$

This can be written

$$
B=F\left(Z_{2}\right)-I\left(Z_{1}\right)+e^{2} /\left(r_{1}+r_{2}\right)
$$

where the electron affinity of the atom of atomic number $Z_{2}$, namely the energy released when an electron is added to the atom, is

$$
F\left(Z_{2}\right)=E\left(Z_{2}, Z_{2}\right)-E\left(Z_{2}+1, Z_{2}\right)
$$

and the ionization energy $I$ of atom 1 , the energy required to remove an electron, is

$$
I\left(Z_{1}\right)=-E\left(Z_{1}, Z_{1}\right)+E\left(Z_{1}-1, Z_{1}\right) .
$$

Which atom donates an electron and which receives is determined by maximizing the binding energy. In general, the atom of lower $Z$ is the donor atom. In eq.4.27 we have estimated the equilibrium separation of the two ions as the sum of the mean radii since the overlapping electron clouds will inhibit closer approach. Using the sum of the rms radii does not lead to significantly different results. 
In fact, however, we find that, when the binding is positive, the maximum binding comes when the atom of lesser $Z=Z_{1}$ donates all its electrons to the other atom of greater atomic number $Z_{2}$. This does not result in a violation of eq.3.22.

The binding energy is then

$$
B=E\left(Z_{1}, Z_{1}\right)+E\left(Z_{2}, Z_{2}\right)-E\left(0, Z_{1}\right)-E\left(Z_{2}+Z_{1}, Z_{2}\right)+\frac{\alpha Z_{1}^{2}}{r\left(Z_{1}+Z_{2}, Z_{2}\right)}
$$

The mean radius of the totally stripped ion, $r(0, Z)$, is taken to be zero. The molecular binding energy for hydrogen with an element of atomic number $Z$ can, therefore, be expressed as

$$
B=E(Z, Z)-E(Z+1, Z)-13.6 e V+\alpha / r(Z+1, Z) .
$$

This is the electron affinity of the element of atomic number $Z$ minus the hydrogen ionization energy plus the negative of the Coulomb energy of attraction of the two ions.

\begin{tabular}{||l|l|ccc||}
\hline $\mathrm{Z}$ & & $I_{\text {susy }}$ & $I_{\text {exp }}$ & $r_{\text {susy }}$ \\
\hline \hline & & & & \\
1 & $\mathrm{H}$ & 13.6 & 13.6 & 0.792 \\
3 & $\mathrm{Li}$ & 23.8 & 5.4 & 0.023 \\
11 & $\mathrm{Na}$ & 64.6 & 5.1 & 0.0070 \\
19 & $\mathrm{~K}$ & 105 & 4.3 & 0.0041 \\
37 & $\mathrm{Rb}$ & 197 & 4.2 & 0.0021 \\
55 & $\mathrm{Cs}$ & 289 & 3.9 & 0.0014 \\
& & & & \\
\hline
\end{tabular}

Table 1: single electron ionization energies of alkali atoms in $\mathrm{eV}$ in the exact susy case using the optimum wave function from eq.3.16. In the fourth column we give the experimental ionization energies in our broken susy world. The fifth column gives the predicted mean radius of the electron/selectron cloud in Angstroms.

In table 1 we tabulate the ionization energies of the alkali atoms and the mean atomic radii in the susy case to compare with the experimental values in the broken susy case. It is seen that, for large $Z$ in the susy case, the ionization energy is significantly greater than the experimental values in our broken susy world as would be expected since all the electrons and selectrons are in the $1 s$ ground state. In the current universe, all atoms have a radius of order $1 \mathrm{~A}^{\circ}$ since, for greater $\mathrm{Z}$, the increased attraction to the nucleus is balanced by electron repulsion and by the need to put electrons into higher orbitals which have greater spatial extent. In exact susy on the contrary, as can be seen from table 1, heavier atoms have significantly smaller radii. In table 2, we give the electron affinities for the halides, namely the energy released if an extra electron is dropped onto the neutral atom making a negatively charged ion. Table 3 gives the estimated binding energies for the alkali halides in 


\begin{tabular}{||l|l|ll||}
\hline $\mathrm{Z}$ & & $F_{\text {susy }}$ & $r_{\text {susy }}$ \\
\hline \hline & & & \\
9 & $\mathrm{~F}$ & 35.38 & 0.122 \\
17 & $\mathrm{Cl}$ & 146.3 & 0.066 \\
35 & $\mathrm{Br}$ & 669.4 & 0.033 \\
53 & $\mathrm{I}$ & 1571 & 0.022 \\
& & & \\
\hline
\end{tabular}

Table 2: electron affinities in $\mathrm{eV}$ and mean radii in Angstroms of the halide atoms in the exact susy case using the trial wave function from eq.3.16.

\begin{tabular}{||c|cccccc||}
\hline & & & & & & \\
& $\mathrm{H}$ & $\mathrm{Li}$ & $\mathrm{Na}$ & $\mathrm{K}$ & $\mathrm{Rb}$ & $\mathrm{Cs}$ \\
\hline \hline \multirow{3}{*}{$\mathrm{F}$} & 134 & 557 & -.089 & 6610 & 24000 & 44900 \\
& $(5.87)$ & $(5.91)$ & $(5.3)$ & $(5.07)$ & $(5.0)$ & $(5.1)$ \\
$\mathrm{Cl}$ & 93.14 & 1570 & 4140 & -4.5 & 47000 & 106000 \\
& $(4.43)$ & $(4.8)$ & $(4.2)$ & $(4.34)$ & $(4.3)$ & $(4.58)$ \\
$\mathrm{Br}$ & 113.1 & 4680 & 28100 & 41400 & -12.7 & 139000 \\
& $(3.83)$ & $(4.3)$ & $(3.74)$ & $(3.91)$ & $(3.9)$ & $(0.34)$ \\
$\mathrm{I}$ & 134.2 & 8920 & 56300 & 112000 & 94800 & -21.0 \\
& & $(3.5)$ & $(3.0)$ & $(3.31)$ & $(3.3)$ & $(3.56)$ \\
\hline
\end{tabular}

Table 3: Binding energy estimates in eV from eq.4.30 for the alkali halides in the exact susy case using the trial wave function from eq.3.16. Negative entries correspond to combinations with no ionic binding in the susy world although they are bound in the broken susy world. Experimental binding energies in the broken susy case, given in parentheses, are taken from [8]. 
the susy case following eq.4.30. The negative entries correspond to molecules that are not bound in the susy case although they do form diatomic molecules in the broken susy case. Covalent binding of alkali-halides is negligible in the broken susy world and presumably also negligible in the exact susy world at least for those molecules that have significant positive ionic binding energy.

Where the susy binding energies are positive they are much greater than in broken susy and the interatomic radii are much smaller. The smaller radii of the susy atoms suggests that chemical reaction rates will be much slower in the susy world, while the greater molecular binding energies suggests that the diatomic molecules which do bind will be much sturdier than in the broken susy case since the dissociation energies reach well into the gamma ray region. However, these results are moot if, as suggested by the semi-empirical mass formula [2], nuclei above oxygen are rare or nonexistent in a susy background. If there were nonnegligible surviving heavy elements, due to the very large binding energies in table 3 , they would rapidly steal atoms from lighter molecules.

It is also interesting to look at the low-lying diatomic molecules whose susy binding energies in the current approximation are given in table 4. Unlike the case in the broken susy world, in exact susy there is significant binding of helium to other low-lying elements. Molecules with small or negative ionic binding energy could be bound through covalent binding which is not treated in this article. We find no ionic binding in diatomic molecules whose atoms differ by zero or one unit of atomic number except for a few of the lighter molecules such as helium hydride which is only marginally bound in broken susy.

In the case of homonuclear diatomic molecules we can write, following eq.4.26, the ionic binding energy due to the transfer of $l$ electrons from one atom of atomic number $Z$ to the other as

$$
\begin{array}{r}
B=2 E(Z, Z)-E(Z-l, Z)-E(Z+l, Z)+\frac{l^{2} \alpha}{r(Z-l, Z)+r(Z+l, Z)} \\
=-\frac{l^{2} R_{\infty}}{384}\left(79 Z+70+\frac{400 l^{2}}{11 Z+5}\right)
\end{array}
$$

Since this is negative for arbitrary $l$, the homonuclear diatomic molecules have no ionic bonding for the approximate wave functions we are using. Although no ionic bonding is found for these potential molecules, the negative binding energies are small compared to those of the alkali-halides. This suggests that with a better approximation of the wave functions, ionic binding may occur and, additionally, one might expect covalent binding to be effective for these molecules.

\section{$5 \quad$ Supersymmetric water}

As a final example we consider the ionic binding of the susy water molecule. In the current (broken susy) universe, water binds due to a transfer of an electron from each of the two hydrogen atoms to $2 p$ orbitals of the oxygen atom resulting in a closed shell of oxygen. Since 


\begin{tabular}{|c|c|c|c|c|c|c|c|c|c|}
\hline & $\mathrm{H}$ & $\mathrm{He}$ & $\mathrm{Li}$ & $\mathrm{Be}$ & B & C & $\mathrm{N}$ & $\mathrm{O}$ & $\mathrm{F}$ \\
\hline $\mathrm{H}$ & $\begin{array}{c}-1.9 \\
(4.48)\end{array}$ & $\begin{array}{c}11.0 \\
(.003)\end{array}$ & $\begin{array}{c}25.1 \\
(2.43)\end{array}$ & $\begin{array}{c}40.4 \\
(2.03)\end{array}$ & $\begin{array}{c}56.8 \\
(3.42)\end{array}$ & 74.4 & $\begin{array}{c}93.2 \\
(<3.47)\end{array}$ & $\begin{array}{l}113.1 \\
(4.39)\end{array}$ & $\begin{array}{l}134.2 \\
(5.87)\end{array}$ \\
\hline $\mathrm{He}$ & & $\begin{array}{c}-16.5 \\
(0.0009)\end{array}$ & 27.6 & 74.1 & 123 & 174 & 227 & 283 & 341 \\
\hline $\mathrm{Li}$ & & & $\begin{array}{l}-57.1 \\
(1.4)\end{array}$ & 36.4 & 133 & 234 & 338 & $\begin{array}{c}446 \\
(3.49)\end{array}$ & $\begin{array}{c}557 \\
(5.91)\end{array}$ \\
\hline $\mathrm{Be}$ & & & & -137 & 23.9 & 190 & 360 & $\begin{array}{c}536 \\
(4.6)\end{array}$ & $\begin{array}{c}716 \\
(5.8)\end{array}$ \\
\hline B & & & & & $\begin{array}{l}-270 \\
(3.0)\end{array}$ & $\begin{array}{r}-23.2 \\
(4.6)\end{array}$ & 230 & $\begin{array}{c}489 \\
(8.28)\end{array}$ & $\begin{array}{c}754 \\
(7.8)\end{array}$ \\
\hline C & & & & & & $\begin{array}{c}-470 \\
(6.21)\end{array}$ & $\begin{array}{l}-118 \\
(7.7)\end{array}$ & $\begin{array}{c}240 \\
(11.0)\end{array}$ & 606 \\
\hline $\mathrm{N}$ & & & & & & & $\begin{array}{c}-749 \\
(9.76)\end{array}$ & $\begin{array}{c}-275 \\
(6.50)\end{array}$ & $\begin{array}{c}207 \\
(3.5)\end{array}$ \\
\hline $\mathrm{O}$ & & & & & & & & $\begin{array}{l}-1121 \\
(5.12)\end{array}$ & -507 \\
\hline $\mathrm{F}$ & & & & & & & & & $\begin{array}{c}-1600 \\
(1.60) \\
\end{array}$ \\
\hline
\end{tabular}

Table 4: Binding energy estimates in eV from eq.4.30 for the low lying diatomic molecules in the exact susy case using the trial wave function from eq.3.16. Negative entries correspond to unbound combinations. Experimental binding energies [8] in the broken susy world are given in parentheses for the molecule indicated by the line above. 
the $p$ orbitals are in one to one correspondence with the Cartesian coordinates, the hydrogen bonds, in zeroth approximation, are at right angles to each other. Coulomb repulsion of the protons then leads to the slightly greater observed bond angle of $105^{\circ}$.

In the susy case, all the electrons can be put into the spherically symmetric $1 s$ wave function of oxygen, converting, if necessary, into scalar electrons via eq.1.1. Proton repulsion will then naturally lead to a linear triatomic molecule with the oxygen ion in the center. Since this arrangement has no molecular dipole moment, the properties of water as a solvent might be significantly changed in susy matter.

In this linear configuration, the binding energy of a neutral atom of atomic number $Z$ with two hydrogen atoms would be

$$
\begin{aligned}
B=E(Z, Z)-E(Z & +2, Z)-2 R_{\infty}+\frac{3 \alpha}{2 r(Z+2, Z)} \\
& =\frac{R_{\infty}}{128}\left(11 Z^{2}+66 Z-311\right)
\end{aligned}
$$

For $\mathrm{H}_{2} \mathrm{O}$ this corresponds to a total dissociation energy of $98 \mathrm{eV}$. Atoms lighter than beryllium will not have a positive ionic binding energy to two hydrogen atoms.

\section{Conclusions}

The variational approach we have taken in this paper allows for an approximate but analytic expression for the atomic energies, mean radii, and ionic molecular binding energies as a function of the electron charge and mass. We have tabulated numerical results for the case where the degenerate electron/selectron mass is equal to the electron mass in the broken susy world but, obviously, other masses can be used if that becomes theoretically motivated. Our core assumptions are that, in the susy background as in our world, the electron mass is non-zero but much less than the nucleon mass, the nucleus is small compared to atomic scales, and atomic structure is governed by a $U(1)$ electromagnetism. The results of the paper are also independent of any assumptions about atomic abundances as long as these are non-zero. Of course, the degenerate susy masses and the abundances would be important parameters in discussing the transition to exact susy and the subsequent properties of the susy world.

Our approximate atomic wave function corresponds to putting all electrons into a screened $1 s$ wave function. This approximation is of limited usefulness in the broken susy world for atoms above helium due to the Pauli principle but could be a good approximation in the exact susy world. We have neglected mixing between atoms with the same $Z$ but different numbers of scalar electrons. We have computed ionic binding energies of various atoms assuming that atoms remaining spherically symmetric is a reasonable zeroth approximation as in the broken susy world. We have also assumed that the bond lengths are approximately the sum of the mean atomic radii. This assumption is found to be reasonable in broken susy. We have treated the atomic ions quantum mechanically but the molecular bound states 
are treated classically. A similar semi-classical calculation gives reasonable results for the alkali-halides in the broken susy world.

As expected, we find that the atomic sizes are quite small in general compared to the corresponding atomic sizes in the broken susy world. This tends to lead to large ionic binding energies since the transfer of an electron from one atom to another leads to a strong Coulomb attraction. Such molecules could be expected to be much sturdier in a susy world. On the other hand some of the heavier alkali halides that are bound in the broken susy world become unbound in exact susy. This is due to the higher ionization energy of the heavy alkali atoms in exact susy where all the electrons are in the $1 s$ wave function.

It is interesting to inquire whether the diatomic molecules that are unbound in the susy world play any essential roles in the broken susy world. This might lead to an anthropic explanation of why our world is not exactly supersymmetric. In fact however, from an environmental point of view one readily comes on reasons to welcome the absence of a few of the molecules that are unbound in the susy universe but have severe deleterious effects in our broken susy world. For example, among the low-lying diatomic molecules, sodium fluoride, $\mathrm{NaF}$, potassium chloride, $\mathrm{KCl}$, and potassium fluoride, $\mathrm{KF}$, are known to have lifethreatening effects in the broken susy world. Of these, $\mathrm{NaF}$ and $\mathrm{KCl}$, are seen in table 3 to be unbound in the exact susy world.

The susy water molecule is predicted to be linear rather than forming the familiar $105^{\circ}$ bond angle seen in the broken susy world. The resulting absence of a dipole moment could be significant with respect to viability since, in the broken susy world, the anomalously large dipole moment of the water molecule is important in making water a universal solvent facilitating chemical reactions.

Clearly, we have barely begun to explore the properties of bulk susy matter and we are far from being able to make any statements about the possibility of life in the susy world. In the more proximate future it should be possible to explore the effects of mixing between states of differing selectron numbers and to estimate covalent binding energies as well as to seek an improved variational wave function.

\section{Acknowledgements}

This work was supported in part by the US Department of Energy under grant DE-FG0296ER-40967. We acknowledge helpful discussions with R. Tipping.

\section{References}

[1] Steven Giddings, hep-th/0303031, Phys. Rev. D68,026006 (2003).

[2] L. Clavelli, IJMPE 15, 1157 (2006),hep-th/0508207; hep-ph/0607029 talk presented at Susy06, Irvine CA, published in condensed form in the 14th Int. Conf. on Susy and the Unification of Fundamental Interactions, ed. J. Feng, AIP, 2007. 
[3] P. Frampton, hep-th/0302007; Mod.Phys.Lett. A19, 801 (2004); Mod. Phys. Lett. A21, 649 (2006), hep-th/0508082 .

[4] L. Clavelli and G. Karatheodoris, Phys. Rev. D72, 035001 (2005).

[5] L. Clavelli, hep-ph/0602024, Journal of High Energy Density Physics, HEDP 2006.06.002.

[6] C. Eckart, Phys. Rev. 36, 878 (1930)

[7] L. Pauling and E.B. Wilson, Introduction to Quantum Mechanics, McGraw-Hill, New York (1935).

[8] K.P. Huber and G. Herzberg, Constants of Diatomic Molecules Van Nostrand Reinhold, New York (1979). 